\title{
Iterative Error Diffusion Halftoning Using a Zero Phase Error Filter
}

\author{
Anamitra Makur, Akhil Kumar* \\ Department of Electrical Communication Engineering \\ Indian Institute of Science \\ Bangalore, India
}

\begin{abstract}
The halftone quality of an error diffusion halftone depends on the error filter response. Apart form the magnitude response being a low pass, it is desirable to have a zero phase response. In this paper we propose the iterative error diffusion halftoning algorithm that allows use of non-causal error filter (such as zero phase error filter). We present simulation results showing that the proposed halftoning scheme is substantially better, specially in retaining the sharpness of the edges and in truthfullness.
\end{abstract}

\section{Introduction}

Error diffusion [1] is regarded as a breakthrough in digital image halftoning. The solid lines in Figure 1 show the block schematic of error diffusion. The input to the quantizer $y_{m}(i, j)=f(i, j)+\left\{h^{+}(k, l) * e_{m}(i, j)\right\}$ is 1-bit quantized to a halftone $g_{m}(i, j)$, where $*$ denotes convolution. The two components of the input are $f(i, j)$, the input gray value, and the filtered (diffused) error. The quantization error $e_{m}(i, j)=$ $y_{m}(i, j)-g_{m}(i, j)$ is filtered through an error flter $h^{+}(k, l)$ to obtain this component. The error filter is causal FIR with $h^{+}(0,0)=0$ such that a sequential single-pass algorithm results.

The performance of error diffusion depends primarily on the error filter. It is recognized that the magnitude response of the error filter should be low pass, since this would result in the low frequency spectrum of the halftone resembling the image spectrum. We further recognize that the error filter should preferably be zero phase, so that the error feedback is added to the input image in phase to retain the sharp edges. Some earlier work on halftoning provides indirect reference to phase response; examples are [2] in the context of error diffusion, and [3] in the context of iterative Fourier transform halftoning. We propose here a modification to the error diffusion halftoning algorithm that may use a zero phase error filter and improve the quality of the output.

\footnotetext{
*Currently with Tata-IBM Limited, Bangalore, India
}

\section{Iterative error diffusion}

Since a zero phase error filter is not causal, it is not possible to be realized in a single-pass algorithm. We propose an iterative error diffusion (multi-pass) where the quantization error of one iteration (pass) is collected and used during the next iteration as quantization error of the future pixels. Figure 1 (both solid and dashed lines) shows one iteration of the iterative error diffusion. The error filter $h(k, l)$ is realized using two parts, a strictly causal part $h^{+}(k, l)$ and the remaining part $h^{-}(k, l)$. The subscript $m$ denotes the iteration number. The quantization error is convolved with $h(k, l)$ and added to the input image. While past quantization errors from the same iteration, $e_{m}(i, j)$, are used by $h^{+}(k, l)$, quantization errors taken from the previous iteration, $e_{m-1}(i, j)$, are used by $h^{-}(k, l)$. Thus, the input to the quantizer in the $m$ th iteration is

$$
\begin{aligned}
y_{m}(i, j)= & f(i, j)+\left\{h^{+}(k, l) * e_{m}(i, j)\right\} \\
& +\left\{h^{-}(k, l) * e_{m-1}(i, j)\right\} .
\end{aligned}
$$

Since the error filter $h(k, l)$ may be non-causal, a zero phase error filter may be used with the iterative error diffusion halftoning.

Now we derive an expression for the halftoning noise spectrum. Since $e_{m}(i, j)=y_{m}(i, j)-g_{m}(i, j)$, it follows that

$$
\begin{aligned}
E_{m}\left(\omega_{x}, \omega_{y}\right)= & F\left(\omega_{x}, \omega_{y}\right)-G_{m}\left(\omega_{x}, \omega_{y}\right) \\
& +H^{+}\left(\omega_{x}, \omega_{y}\right) E_{m}\left(\omega_{x}, \omega_{y}\right) \\
& +H^{-}\left(\omega_{x}, \omega_{y}\right) E_{m-1}\left(\omega_{x}, \omega_{y}\right) .
\end{aligned}
$$

where $E_{m}, E_{m-1}, F, G_{m}, H^{+}$and $H^{-}$are the Fourier transforms of $e_{m}, e_{m-1}, f, g_{m}, h^{+}$and $h^{-}$in that order. The halftoning noise is the difference between the input and the halftone,

$$
n_{m}(i, j)=f(i, j)-g_{m}(i, j)
$$

in the $m$ th iteration. If $N_{m}$ denotes its Fourier spectrum, then

$$
\begin{aligned}
N_{m}\left(\omega_{x}, \omega_{y}\right)= & \left(1-H^{+}\left(\omega_{x}, \omega_{y}\right)\right) E_{m}\left(\omega_{x}, \omega_{y}\right) \\
& -H^{-}\left(\omega_{x}, \omega_{y}\right) E_{m-1}\left(\omega_{x}, \omega_{y}\right) .
\end{aligned}
$$


If the algorithm converges after $M$ iterations then

$$
E_{m}\left(\omega_{x}, \omega_{y}\right) \approx E_{M}\left(\omega_{x}, \omega_{y}\right) \quad \forall m \geq M .
$$

This leads to

$$
N_{m}\left(\omega_{x}, \omega_{y}\right)=E_{M}\left(\omega_{x}, \omega_{y}\right)\left(1-H\left(\omega_{x}, \omega_{y}\right)\right) \quad \forall m \geq M
$$

where $H$ is the transfer function of the error filter. This is identical to what happens in conventional error diffusion [4].

In practice, a convergence is achieved when a resulting halftone has no perceptual difference from the previous halftone. At convergence, let the difference between two consecutive halftones be $\tilde{n}_{m}(i, j)=$ $g_{m-1}(i, j)-g_{m}(i, j)$. A perceptual convergence occurs when the halftoning noise spectrum changes by some fixed amount during each iteration after $M$ iterations,

$$
E_{m}\left(\omega_{x}, \omega_{y}\right)-E_{m-1}\left(\omega_{x}, \omega_{y}\right) \approx \tilde{E}\left(\omega_{x}, \omega_{y}\right) \quad \forall m \geq M .
$$

It follows that

$$
\tilde{N}_{m}\left(\omega_{x}, \omega_{y}\right)=\tilde{E}\left(\omega_{x}, \omega_{y}\right)\left(1-H\left(\omega_{x}, \omega_{y}\right)\right) \quad \forall m \geq M
$$

where $\tilde{N}_{m}$ is the Fourier spectrum of $\tilde{n}_{m}$. Since $(1-$ $\left.H\left(\omega_{x}, \omega_{y}\right)\right)$ has a high pass filter response, after a few initial iterations, the change in halftones is limited to high frequency regions shaped by $1-H\left(\omega_{x}, \omega_{y}\right)$, and therefore perceptually unimportant.

One advantage of a zero phase error filter is observed from the above expression. If $H\left(\omega_{x}, \omega_{y}\right)$ is a zero phase (real) low pass, then $1-H\left(\omega_{x}, \omega_{y}\right)$ is a zero phase high pass filter having complementary magnitude response to that of $H\left(\omega_{x}, \omega_{y}\right)$. However, if the error filter is not zero phase (complex response), $1-H\left(\omega_{x}, \omega_{y}\right)$ is a nonzero phase high pass whose magnitude response is no longer an exact complement of the error filter magnitude response. Thus, while in general both the magnitude and the phase response of the error filter decides the noise spectrum (which is not easy to determine), in case of zero phase error filters only the magnitude response decides the noise spectral shape (which is just its complement).

The iteration is initialized with an error image $e_{0}(i, j)$, containing some starting quantization error for all pixels. We have observed that the starting set of quantization error values, if taken from the quantization error of any actual halftoning algorithm (than, say, a random noise), achieves faster convergence. The quantization error is updated on the error image on a pixel-by-pixel basis. The error filter we use is a zero phase (opposite quadrant symmetry) low pass filter with unity gain at zero frequency, derived from the desired shape of the halftoning noise. $h(0,0)$ should

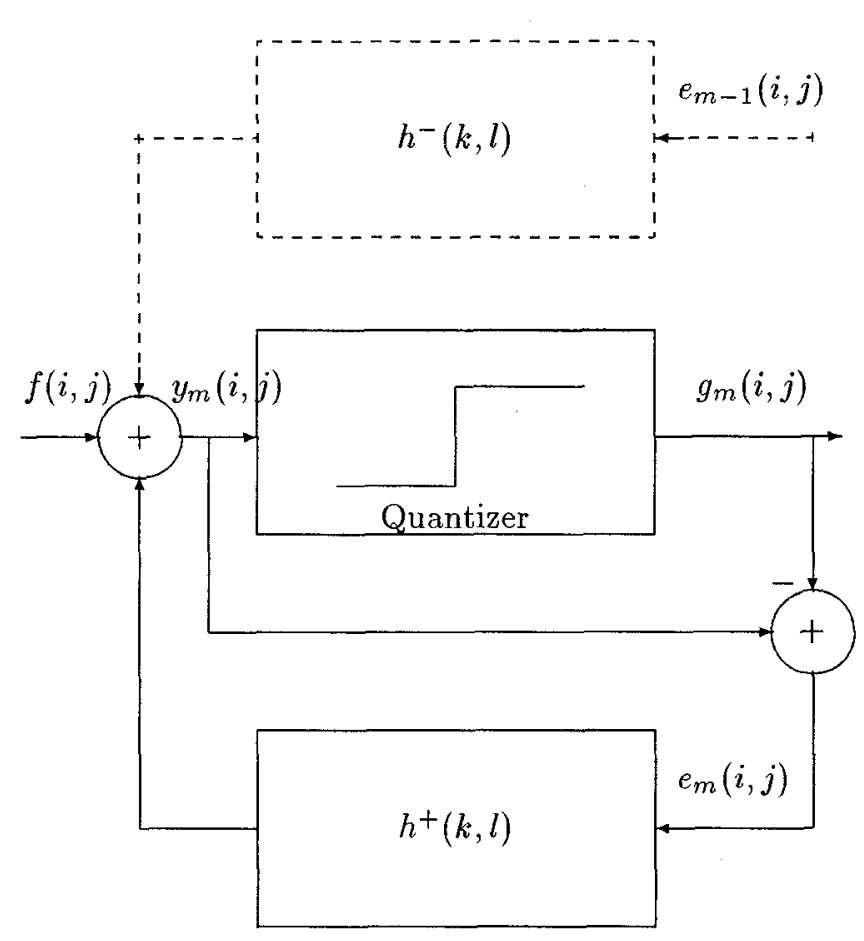

Figure 1: Schematic for conventional and iterative error diffusion

be zero, since having a nonzero $h(0,0)$ means total error is not diffused, leading to a halftoning noise having considerable low frequency energy. The iterations are continued until a convergence criterion is satisfied. The convergence criterion used in our iterative error diffusion is the energy of the difference between two consecutive halftones, which is compared with a threshold.

\section{Simulation results}

Figure 2 shows the halftone obtained from the conventional error diffusion using the following $3 \times 5$ error filter:

$$
\begin{aligned}
& h(k, l)= {\left[\begin{array}{lllll}
.00 & .00 & .00 & .15 & .10 \\
.06 & .10 & .15 & .10 & .06 \\
.03 & .06 & .10 & .06 & .03
\end{array}\right] } \\
& 0 \leq k \leq 2, \quad-2 \leq l \leq 2
\end{aligned}
$$

Figure 3 shows the halftone obtained after a single iteration of iterative error diffusion using error diffusion errors as the starting error values, with the following $5 \times 5$ zero phase error filter (derived from the earlier 


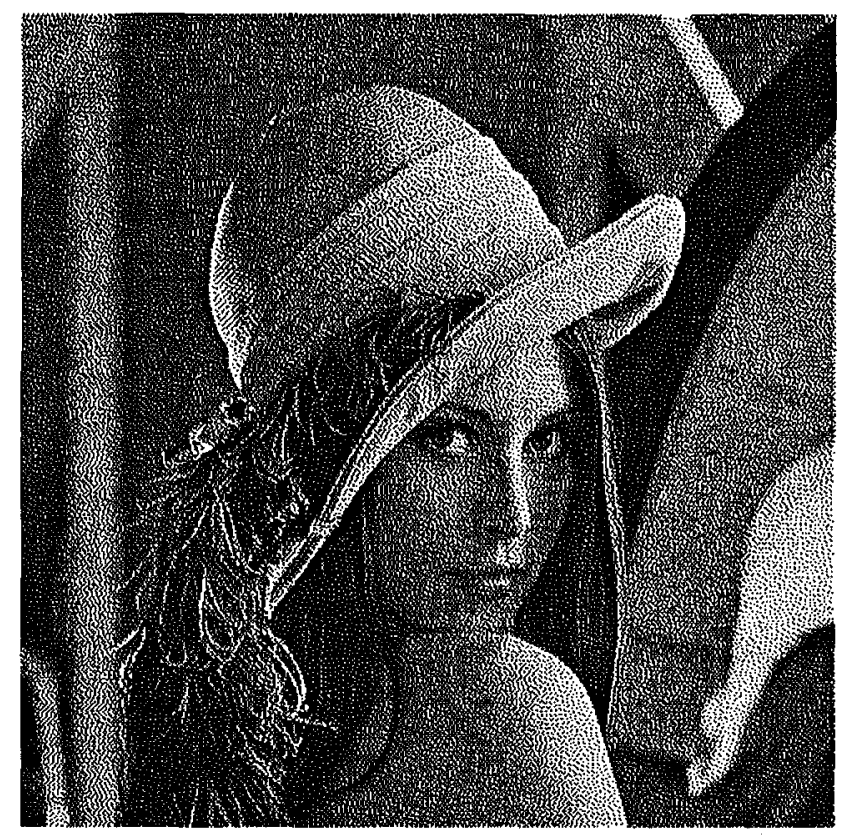

Figure 2: Conventional error diffusion

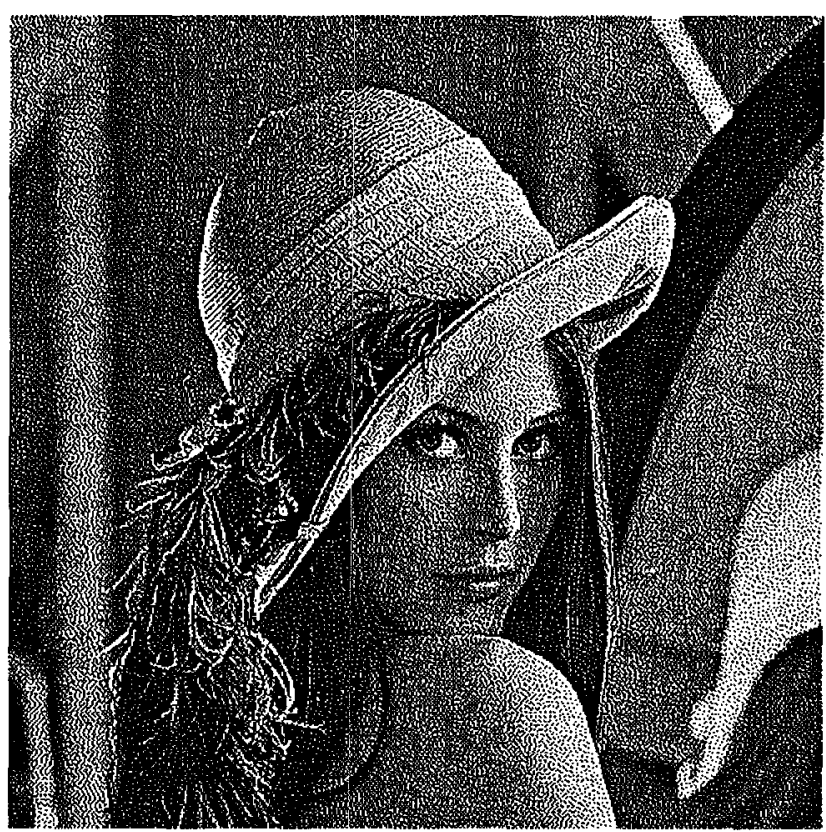

Figure 3: Iterative error diffusion, after 1 iteration

error filter):

$$
\begin{aligned}
& h(k, l)=\left[\begin{array}{lllll}
.015 & .030 & .050 & .030 & .015 \\
.030 & .050 & .075 & .050 & .030 \\
.050 & .075 & .000 & .075 & .050 \\
.030 & .050 & .075 & .050 & .030 \\
.015 & .030 & .050 & .030 & .015
\end{array}\right] \\
& -2 \leq k \leq 2, \quad-2 \leq l \leq 2 .
\end{aligned}
$$

Figure 4 shows the halftone obtained after 10 iterations. Observe that even a single pass of the iterative error diffusion produces superior results. All the regions which have fine details, such as the lines and folds in the hat (which were all missing in figure 2), are very clear in figure 4 . Zero phase error filter is more truthful in reproducing the fine details of the actual image.

Figures 5 and 6 show the halftoning noise magnitude spectrum for the halftones of figure 2 and 4 . While the error diffusion noise spectrum is not high pass in all directions due to nonzero phase nature of the error filter, the iterative error diffusion noise spectrum takes the shape of the complement of the error filter magnitude response in accordance to our derivation. Figure 7 compares the radially averaged absolute phase error for three halftones. Consistently low level of phase error is obtained by the zero phase error filter.

Phase response of the error filter in error diffusion halftoning is important for the halftone quality, and

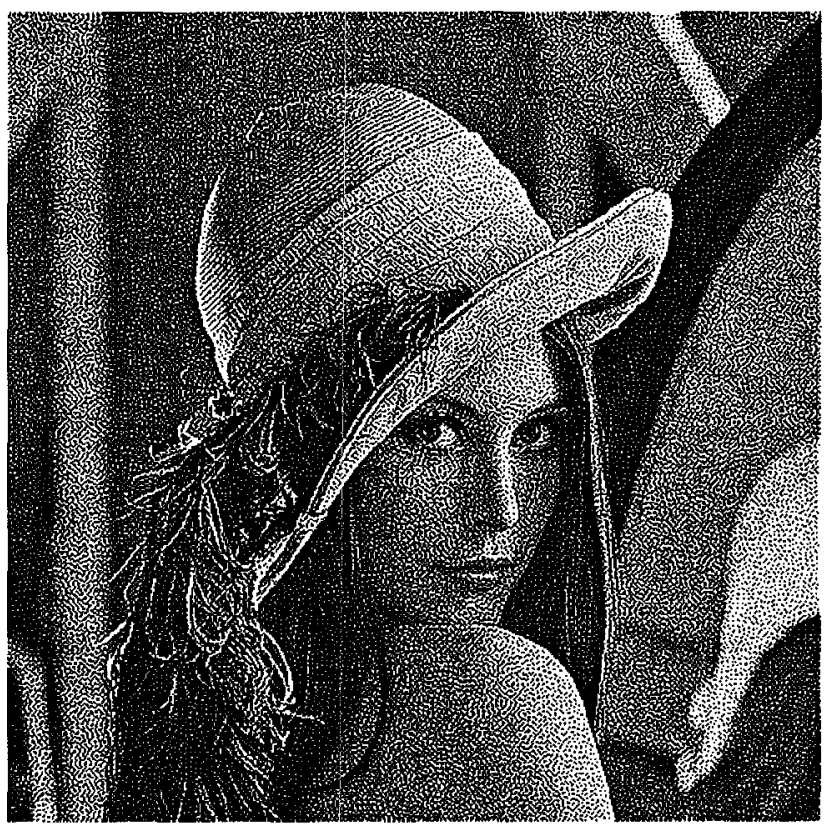

Figure 4: Iterative error diffusion, after 10 iterations 


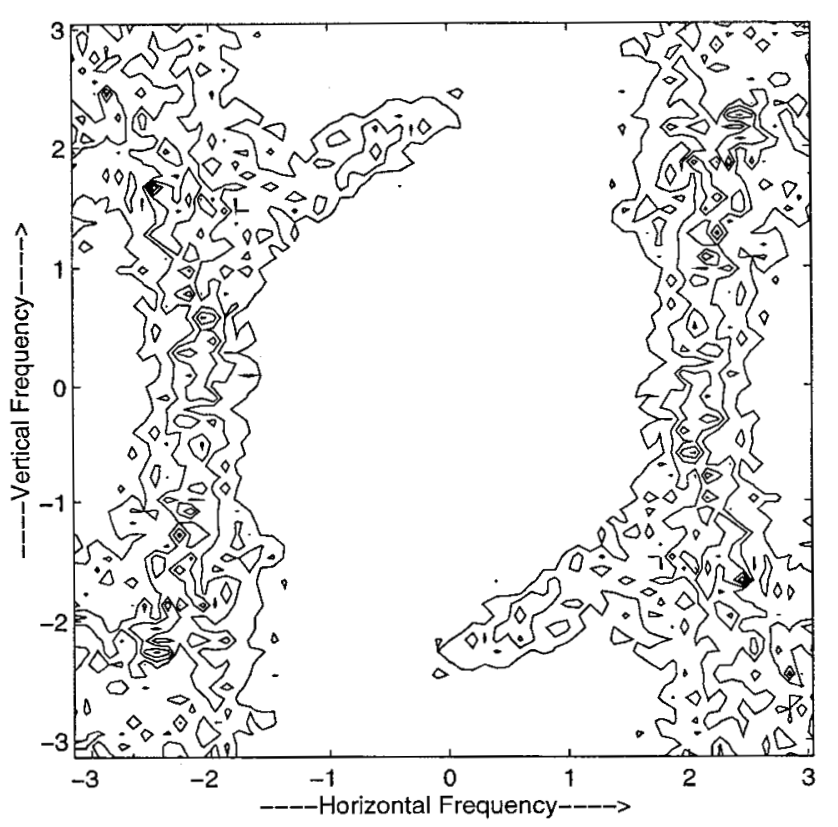

Figure 5: Halftoning noise for figure 2

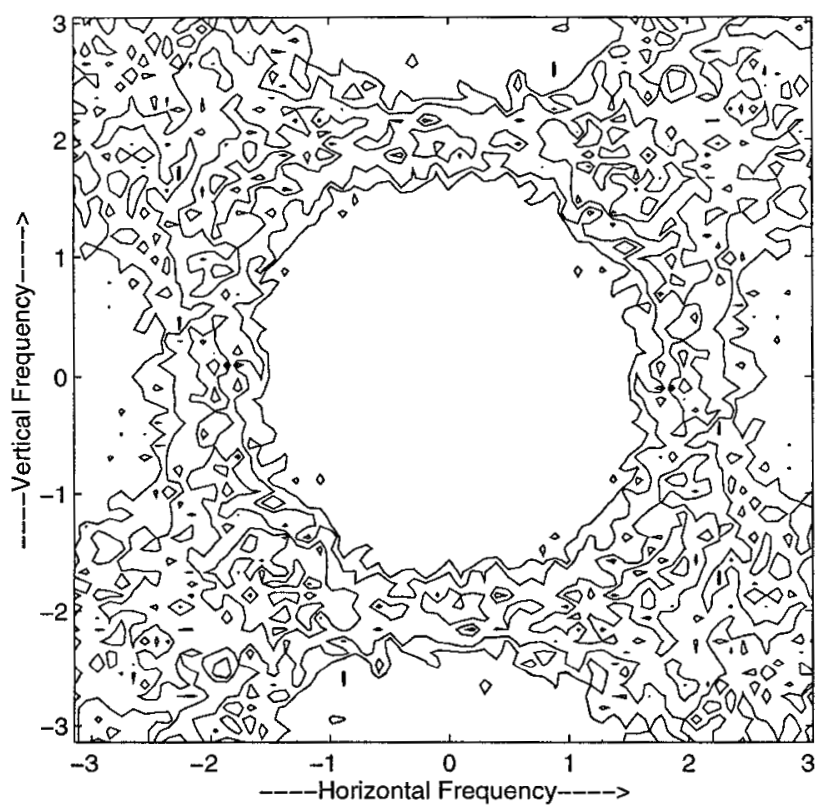

Figure 6: Halftoning noise for figure 4

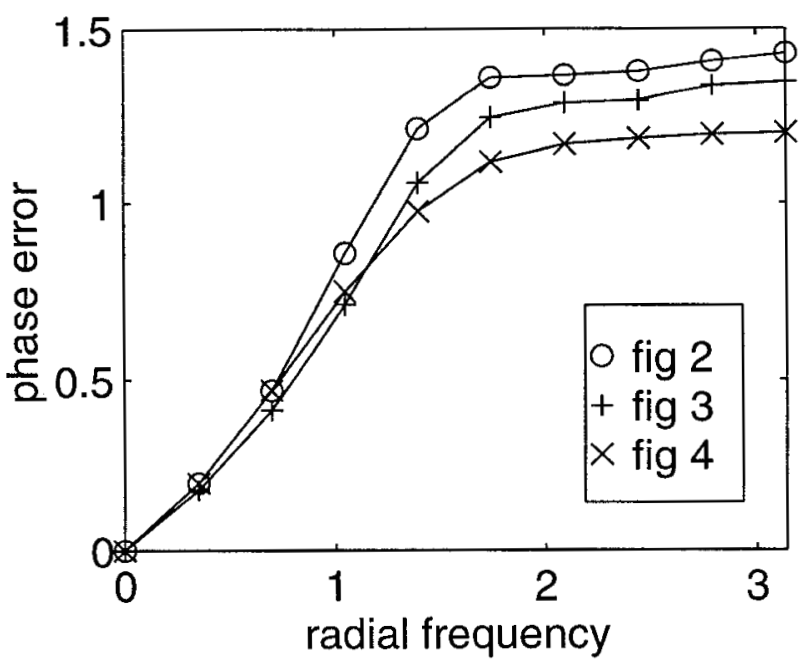

Figure 7: Phase errors of halftones

should ideally be zero phase. We have proposed an iterative error diffusion halftoning algorithm that allows use of non-causal error filter. Thus, zero phase error filters may be used with the proposed algorithm. From the simulation results, we conclude that iterative error diffusion using a zero phase error filter produces substantially better halftones that retains all the edges and their sharpness. Few iterations are needed for perceptual convergence, and even a fixed number of iterations (say, one) improves the halftone quality.

\section{References}

[1] R. W. Floyd and L. Steinberg, "An adaptive algorithm for spatial greyscale," Proc. Soc. Inf. Disp, Vol.17, pp.75-77, 1976.

[2] S. Kollias and D. Anastassiou, "A unified neural network approach to digital image halftoning," IEEE. Trans. Sig. Proc, Vol.39, pp.980-984, Apr.1991.

[3] M. Broja, F. Wyrowski and O. Bryngdahl, "Significance of spectral phase in synthesis of binarized images," Opt. Commun., Vol.95, pp.205-209, 1993.

[4] A. Kumar, "Some improvements in error diffusion halftoning of images," Master's project report, Department of Electrical Communication Engineering, Indian Institute of Science, Jan. 1996. 